\title{
DATA COMPRESSION TECHNIQUE FOR CLOUD STORAGE IN MULTI-USER ENVIRONMENTS
}

\author{
MANINDRA T, PRADEEP KV
}

${ }^{1}$ Department of Computer Science Engineering, VIT University, Chennai, Tamil Nadu, India. Email: tsn.manindrababu2015@vit.ac.in Received: 23 January 2017, Revised and Accepted: 03 March 2017

\begin{abstract}
In many associations, the cloud storages contain a copy (duplicates) of information. For example, the same file would be spared in various locations by different users; too many documents that are not differentiable may in any case duplication a great part of the same data. This consumes lots of memory in cloud storage. Deduplication is a kind of technique used to eliminate duplicate entries on cloud storage. It helps us to remove the duplicate entries of these additional duplicates, by maintaining only one duplicate of the information and giving ownership rights to the alternate duplicates with pointers that lead back to the first duplicate. Organizations much of the time utilizes this technique in cloud storage for many applications, yet it can be effectively utilized to free up space in vital situations also. To allow an end-user to have the ownership of the file if the file is already existing and should terminate the uploading process to the cloud storage server
\end{abstract}

Keywords: Deduplication, Proof of storage, Homo-Morphic Authentication (HAT).

(C) 2017 The Authors. Published by Innovare Academic Sciences Pvt Ltd. This is an open access article under the CC BY license (http://creativecommons. org/licenses/by/4. 0/) DOI: http://dx.doi.org/10.22159/ajpcr.2017.v10s1.19570

\section{INTRODUCTION}

Cloud computing gives you a chance to give virtual capacity to the clients. It primarily helps for little and medium scale commercial enterprises to decrease their consumption of support of capacity servers. Client data put away at cloud should be secured against potential interlopers. Cloud computing is widely used in IT industry. Virtualization, distributed computing and so on integrates the cloud computing. The cloud computing reduces the storage resource and networking results lowcost maintenance for running a company [1] and it is growing very fast with the IT industry worldwide. Even though there are many advantages of cloud computing, but the customers are not showing much interest on using this service because of data security at cloud storage [2].

In cloud environment, deduplication is a process of data compressing to eliminate duplicate copies of repeated data. It optimizes the usage of memory needs by removing the repetitive information. One and only one of a kind occurrence of the information is really held on capacity media, for example, plate or tape. Repetitive information is removed and points with a pointer to the one of kind information duplicate. For instance, an email framework may contain 100 occurrences of the same one megabyte (MB) document connection. On the off chance that the email stage is moved down or chronicled, each of the 100 cases is spared, requiring $100 \mathrm{MB}$ storage room. With information deduplication, one and only case of the connection is really put away; each consequent case is simply referenced back to the one spared duplicate. In this illustration, a 100 MB memory could be lessened to one and only MB. Deduplication allows to remove the duplicate entries of these additional duplicates by sparing only one duplicate of the information and giving ownership to the alternate duplicates with pointers that lead back to the first duplicate. Organizations much of the time utilizes deduplication in cloud storage in many applications, yet it can be utilized to free up space in essential situations also.

Distributed computing is a developing on request, web-based framework. It gives assortment of administrations over web, for example, stockpiling of information, programing and equipment. Today cloud benefits supplier gives stockpiling and assets with ease. Too aware of that the end goal is to dispose of copy duplicates data [3] duplication can be utilized, which reduces the usage storage room. The duplications system is exceptionally helpful however the securities and its protection of the clients are defenseless to both inside and untouchable assault. So as to beat these disadvantages, every client is given their own benefits through approved similarity check and it is being finished half cloud engineering. The design incorporates proprietor to their client, the general population cloud. In the important encryption, the general population and private cloud and the client who is transferring the document into the cloud would be clarified, yet on account of many like approved check public and private cloud is utilized. On account of private and public cloud with the past system does not give secure deduplication as it is influenced by different pariah assaults.

Deduplication offers different advantages. Bring down storage room prerequisites will spare cloud storage consumptions. The more effective utilization of storage space likewise takes into account longer plate maintenance periods, which gives better storage utilization for a more drawn out time and decreases the requirement for tape reinforcements. We propose and utilize the principal of proficient development of duplicatable files proof of storage, which proposes boundless number of confirmations more and redesigning operations. The security of this development will be demonstrated in an irregular way; what's more, the execution will be done tentatively. Information deduplication additionally reduces the information that should be sent over a LAN with remote reinforcements, replicas, and debacle recuperation. In real practice, information deduplication is regularly utilized as a part of conjunction with different types of information diminishment, for example, ordinary replication taken together, these three procedures can be exceptionally powerful at upgrading the utilization of storage room.

Information deduplication [4] is one of the essential systems for removing out multiple duplicates of rehashed data, and it is been widely used in the area of cloud storage technologies to lessen the amount of space used for storage of data. To secure the secrecy of information differential approved copy check is utilized. Unique in relation to the customary duplication framework, diverse benefits of the client are considered other than the information itself. We introduce this approved copy check cloud storage. The half and half cloud design proposes about both people, in general, cloud storage and the public cloud storage. The private/personal cloud assumes an imperative part in our framework, also the privacy and security of this framework is very less, and as the public billow of this system is not much secured, unapproved accessing of the information bringing about security. Keeping in mind the end goal to provide much more secure environment, the personal cloud is given high-level validation. As proposed, we are 
examined the current deduplication framework is nothing but people in public cloud and general cloud. As we demonstrate that our framework is more secure and gives classification to information. In this paper, the detailed view of our proposing framework would be clarified alongside design, information stream with calculations.

Data are a standout among the most important properties while a client outsourcing its documents to distributed storage. Clients ought to be persuaded that the documents put away in the server are not altered. Conventional strategies for securing information uprightness, for example, message validation codes and advanced marks, oblige clients to download the greater part of documents from cloud storage to check, which acquires a substantial correspondence data. The above type of systems will not be appropriate for distributed storage environment administrations where clients can check the trustworthiness as often as possible, for example, consistently. Subsequently, scientists presented proof of the storage to check the uprightness without copying documents from cloud storage [5]. Besides, clients may likewise require a few element operations, for example, alteration, addition, and cancellation, to upgrade their documents, while keeping up the capacity PoS is proposed for such element updating. Conversely with proof of storage utilizes verified structures, for example, the Merkles tree.

This procedure creates a system which gave assurance against word reference assault, yet at the same time, the result will be a loss of security in data. The cloud deduplication system which is suggested gives secure and productive administrations to the deduplications in the square level. Here, it utilizes focalized encrypted and an extra encrypted operation alongside gets to control component. The united encrypted method to meet the prerequisite, two of them is encryption and deduplications method. In this key is determined with encoded in light of the information gave. The basic technique for duplication is that information transferred by various clients will be put away just as if the substance of the information is comparative. The key is aftereffect of the hashing technique of the information portion. The CE brings about the word reference assault, which an assailant figured out how t to, create a key in private cloud [6]. By contrasting the two-figure content, it figures out how to get the record points of interest.

\section{PROPOSED METHODOLOGY}

The primary commitments of this paper are as per the following:

- With our references and knowledge, this was initiative work to present a technique known as deduplication mechanism. Confirmation with storage system (deduplicatable files PoS), that will be settled the structures of differing types and private labeled challenges.

- Compared to the current validated structures, For example Merkles tree, we outline a method validated structures called homomorphic validated trae (HAT); the verification of capacity stage as well as the deduplication stage with comparable calculation storage usage. Deduplication confirmations, dynamical operations, and multi-user deduplications with great consistency.

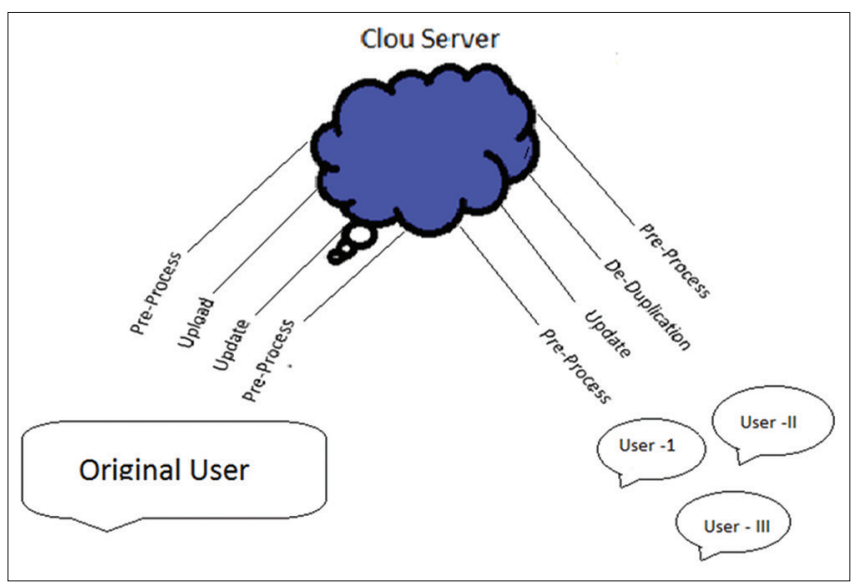

- We are proposing and utilizing the principal of proficient development of duplicatable files proof of storage, which proposes boundless number of confirmations more and redesigning operations. The security of this development will be demonstrated in an irregular way; what's more, the execution will be done tentatively.

- The more effective utilization of storage space likewise takes into account and long maintenance periods, which gives better utilization for a more drawn out time and decreases the requirement for tape reinforcements. Information deduplications additionally lessen the information's that must be sent over a WAN for remote reinforcements and replication.

- Deduplication offers different advantages. Bring down storage room prerequisites will spare cloud storage consumptions. The more effective utilization of storage space likewise takes into account longer plate maintenance periods, which gives better storage utilization for a more drawn out time and decreases the requirement for tape reinforcements.

The following are five stages in the de-duplicatable mechanism framework: Pre-handle, transfer, overhaul, deduplication, confirmation with capacity.

\section{SYSTEM MODEL}

The basic technique for duplication is that information transferred by various clients will be put away just once the substance of information is comparative with the cloud deduplication system which is proposed gives secured and productive administrations to the proposed deduplication in the square level. Moreover, it utilizes focalized encryptions with an extra encryptions operation alongside get to control component. The united encryption method meets the prerequisite of both the encryptions and deduplications. In this key is determined and encoded in light of the information gave. The key is aftereffect of the hashing of the information portion. The CE brings about the word reference assault, which an assailant figured out how to, create a key. By contrasting the two-figure content, it figures out how to get the record points of interest. This procedure creates a system which gave assurance against word reference assault, yet at the same time, result in loss of data.

- In the pre-processing stage, client's transfers their documents. The cloud server chooses whether these records ought to will be transferred. On the off chance that the transfer procedures are in all actuality, go into the transfer stages; generally, that will go in the deduplication stages.

- In the transfer/upload stage, the records to be transferred should not exist in the cloud storage system. The first clients encode in documents and transfer those files to the cloud storage.

- In the deduplication stage, the records to be transferred all-prepared existed in the cloud storage. The resulting clients have the documents locally, and the cloud storage server is going to validate the structure of the records [7]. Consequent clients need to be persuaded the cloud storage server that it possesses documents without transferring them to cloud storage server.

In the overhaul stage, clients may alter, embed, or erase a few pieces of the documents. At that point, they upgrade the corresponding part of the encoded documents and the validated structure in the cloud servers, even the first records will not be transferred without anyone else's input.

\section{SIBLINGS SEARCH}

To encourage operations like deduplication on HAT structure, the following algorithms is used. We describe the way look for computation from path (T, L). It will take a HAT T and a piece record $\mathrm{L}$ of an archive with data yields document sets of center points in the root center from path of the $\mathrm{L}^{\mathrm{th}}$ center point with each one of the nodes which identifies with the $\mathrm{L}^{\text {th }}$ square of a particular record. We do extend the way look for computation to supporting multi-path look as Algorithm, where the $\mathrm{i}^{\text {th }}$ center point in T involves $v$. The figuring takes as information a HAT 
and an asked for summary of the piece records, and yields an asked for once-over of the center documents.

The more viable use of storage room similarly considers and long upkeep periods, which gives better usage for a more drawn out time and declines the necessity for tape fortifications. This technique makes a framework which gave confirmation against word reference strike, yet in the meantime result will be loss of security in information [8].

The cloud deduplication framework which is recommended gives secure and profitable organizations to the deduplications in the square level. Here, it uses centered encoded and an additional scrambled operation close by get the opportunity to control part. The unified encoded technique is to meet the essential of both (encryption and deduplications) strategy [9]. In that, the key is resolved and encoded in light of the data gave. The fundamental system for duplication is data exchanged with different customers would be secured similarly as though the substance of the data is near. The key is eventual outcome of the hashing strategy of the data partition. The CE achieves the word reference strike, which an aggressor made sense of how to, make a key. By differentiating the two make sense of substance, it figures how to get the record purposes of intrigue.

\section{ALGORITHM USED}

\section{Procedure SIBLING (U)}

a. $\quad \psi \leftarrow \varnothing, v \leftarrow \sigma \backslash\{1\}, \sigma \leftarrow \emptyset, \mathrm{k} \leftarrow 1$

b. while $u \neq \emptyset \mathrm{v} \sigma \neq \varnothing$ do

c. if $2 \mathrm{k} € v$ then

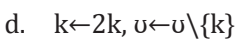

e. if $\mathrm{k}+1 € u$ then

f. $\quad \sigma \leftarrow \sigma U\{(\mathrm{k}+1, \mathrm{FALSE})\}, U \leftarrow u \backslash\{\mathrm{k}+1\}$

g. else

h. $\quad \sigma \leftarrow \sigma \mathrm{U}\{(\mathrm{k}+1, \mathrm{TRUE})\}$

i. else if $2 \mathrm{k}+1 € v$

j. $\quad \mathrm{k} \leftarrow 2 \mathrm{k}+1, U \leftarrow U \backslash\{\mathrm{k}\}, \psi \leftarrow \psi \mathrm{U}\{\mathrm{k}-1\}$

k. else if $u \neq \emptyset$

l. pop out last k insertions $(\alpha, \beta) \mathrm{kn} v$

m. $\mathrm{K} \leftarrow \alpha$

n. if $\alpha=$ FALSE \&\& $\beta=$ TRUE then

o. $\psi \leftarrow \psi U\{\mathrm{k}\}$

p. $\operatorname{return} \psi$

The more effective utilization of storage space likewise takes into account and long maintenance periods, which gives better utilization for a more drawn out time and decreases the requirement for tape reinforcements. Information deduplications additionally lessen the information's that must be sent over a WAN for remote reinforcements and replication [10].

\section{CONCLUSION}

We have proposed a complete solution in the multi-client cloud storage frameworks, and the method of de-duplicatable dynamic proof of storage is presented. We will compose a complete instrument called authentication ownership which is an effective verified structure. In view of this, we proposed the primary commonsense de-duplicatable file proof of storage plot and demonstrated its security in the cloud storage. The hypothetical and test comes about demonstrate that our secure authentication execution is proficient, particularly at the point of the deduplication when the document measures and the quantities of the tested storage technologies.

\section{REFERENCES}

1. Xu W, Feng D, Liu J. Wuhan national lab for optoelectronics, public verifiable proof of storage protocol from lattice assumption. IEEE International Conference on Engineering and Technology (ICETECH); 2015.

2. Hur J, Ko D, Shinn Y, Kang K. Secured data deduplication with Dynamic ownership management in cloud storage. IEEE Trans Knowl Data Eng. DOI: 10.1109/TKDE.2016.2580139.

3. Kaaniche N, Laurent M. SHOPS: Set Homomorphic Proof of Data Possession Scheme in Cloud Storage Applications. Institut Mines-Telecom, Telecom SudParis, UMR CNRS 5157 SAMOVAR. IEEE World Congress on Services; 2015.

4. Li J, Li YK, Chen X, Lee PP, Lou W. A Hybrid cloud approach for secure authorized deduplication. IEEE Trans Parallel Distrib Syst. DOI: 10.1109/TPDS.2014.2318320.

5. Ren Z, Wang L, Wang Q, Xu M. Dynamic proofs of retrievability for coded cloud storage Systems. IEEE Transactions on Services Computing.7. Computer Security Institute, Computer Crime and Security Survey; 2010, 2011. DOI: 10.1109/TSC.2015.2481880.

6. Saritha K, Subasree S. Analysis of hybrid cloud approach for private cloud in the de-duplication mechanism. IEEE International Conference on Engineering and Technology (ICETECH). Coimbatore, TN, India, $20^{\text {th }}$ March; 2015.

7. Xian Z, Waang X, Sun X, Waang Q. A secure and dynamic multi-keywords ranked search schemes over encrypted cloud data. IEEE Trans Parallel Distrib Syst 2016;27(2):340-52.

8. Xiaoo ZJ, Xiao YD. Security and privacy in cloud computing. IEEE Commun Surv Tutor 2013;15(2):843-59.

9. Ardagnaa CA, Asaal R, Daamiani E, Vu QH. From security to assurances in the cloud: A survey. ACM Comput Surv 2015;48(1):201.

10. Ateniese G, Burns R, Curtmola R, Herring J, Kissner L, Peterson Z, et al. Provable data possessions at untrusted stores. In: Proceeding of CCS. 2007. p. 598609 